\title{
Switchable photoluminescence liquid crystal coated bacterial cellulose films with conductive response
}

\author{
Agnieszka Tercjak $^{\mathrm{a}, *}$, Junkal Gutierrez ${ }^{\mathrm{a}}$, Hernane S. Barud ${ }^{\mathrm{b}, \mathrm{c}}$, Sidney J.L. Ribeiro ${ }^{\mathrm{b}}$ \\ a Group 'Materials + Technologies' (GMT), Department of Chemical and Environmental Engineering, Polytechnic School, University of the Basque Country \\ (UPV/EHU), Plaza Europa 1, 20018 Donostia-San Sebastián, Spain \\ ${ }^{\mathrm{b}}$ Laboratory of Photonic Materials, Institute of Chemistry, São Paulo State University - UNESP, Araraquara, SP, Brazil \\ ${ }^{\mathrm{c}}$ Laboratório de Química Medicinal e Medicina Regenerativa (QUIMMERA), Centro Universitário de Araraquara, Araraquara, SP, Brazil
}

\section{A R T I C L E I N F O}

\section{Article history:}

Received 20 October 2015

Received in revised form 5 February 2016

Accepted 6 February 2016

Available online 9 February 2016

\section{Keywords:}

Bacterial cellulose

Nematic liquid crystals

Photoluminescence properties

Conductive properties

UV shielding

\begin{abstract}
A B S T R A C T
Three different low molecular weight nematic liquid crystals (LCs) were used to impregnate bacterial cellulose (BC) film. This simple fabrication pathway allows to obtain highly transparent $\mathrm{BC}$ based films. The coating of $\mathrm{BC}$ film with different liquid crystals changed transmittance spectra in ultraviolet-visible region and allows to design UVC and UVB shielding materials. Atomic force microscopy results confirmed that liquid crystals coated BC films maintain highly interconnected three-dimensional network characteristic of BC film and simultaneously, transversal cross-section scanning electron microscopy images indicated penetration of liquid crystals through the three-dimensional network of $B C$ nanofibers. Investigated $B C$ films maintain nematic liquid crystal properties being switchable photoluminiscence as a function of temperature during repeatable heating/cooling cycles. Conductive response of the liquid crystal coated BC films was proved by tunneling atomic force microscopy measurement. Moreover, liquid crystal coated BC films maintain thermal stability and mechanical properties of the BC film. Designed thermoresponsive materials possessed interesting optical and conductive properties opening a novel simple pathway of fabrication liquid crystal coated BC films with tuneable properties.
\end{abstract}

(c) 2016 Elsevier Ltd. All rights reserved.

\section{Introduction}

Cellulose is one of the most abundant natural polymer which paid increasing attention of many scientists and engineers. Special interest is focused on bacterial cellulose (BC) due to their impressive properties such as high crystallinity, water holding capability, high porosity as well as biodegradability and remarkable biocompatibility (Abeer, Amin, \& Martin, 2014; Almeida et al., 2014; Barud, Caiut, Dexpert-Ghys, Messaddeq, \& Ribeiro, 2012; Fu, Zhang, \& Yang, 2013; Gutierrez, Fernandes, Mondragon, \& Tercjak, 2012; Hoenich, 2006; Iguchi, Yamanaka, \& Budhiono, 2000; Klemm, Schumann, Udhardt, \& Marsch, 2001; Lin et al., 2013; Qiu \& Netravali, 2014; Shi, Zhang, Phillips, \& Yang, 2014; Yano et al., 2005; Zhang et al., 2013; Zhu, Fang, Preston, Li, \& Hu, 2014). These interesting properties of $\mathrm{BC}$, consisted of interconnected three-dimensional nanofibers network, lead to their wide range of application in electronic (Gutierrez, Fernandes, et al., 2012; Lee, Buldum, Mantalaris, \& Bismarck, 2014; Lin et al., 2013; Zhang et al.,

\footnotetext{
* Corresponding author. Tel.: +34 943017 169; fax: +34 943017130

E-mail address: agnieszka.tercjaks@ehu.eus (A. Tercjak).
}

2013, 2014), tissue engineering (Fu et al., 2013; Lin et al., 2013), medicine (Hoenich, 2006; Klemm et al., 2001), drug delivery (Abeer et al., 2014; Almeida et al., 2014) and food packaging (Shi et al., 2014) among others.

Nowadays, many research groups have searched for novel strategies of fabrication composite materials based on BC matrix (Bulota, Tanpichai, Hughes, \& Eichhorn, 2012; Galland et al., 2013; Shaha, Ul-Islama, Khattaka, \& Park, 2013; Zhou et al., 2009). Different type of water-soluble polymeric materials (Barud et al., 2011, 2012; Brown \& Laborie, 2007; Castro et al., 2014; Gea, Bilotti, Reynolds, Soykeabkeaw, \& Peijs, 2010; Hebler \& Klemm, 2009; Marins et al., 2011; Quero et al., 2010; Tercjak, Gutierrez, Barud, Domeneguetti, \& Ribeiro, 2015) or inorganic nanoparticles (Barud et al., 2015; Dal'Acqua et al., 2015; Gutierrez, Fernandes, et al., 2012; Gutierrez, Fernandes, Mondragon, \& Tercjak, 2013; Gutierrez, Tercjak, Argal, Retegi, \& Mondragon, 2012; Jian et al., 2014; Li et al., 2009; Liu, Yang, Wang, Shi, \& Jiang, 2012; Olsson et al., 2010) were employed to develop novel composites with tuneable properties which combine the exceptional properties of BC and physicochemical properties of polymeric materials or optical, electrical, magnetic or antibacterial properties of inorganic nanoparticles. Different preparation pathways such as in-situ biosynthesis, immersion of 
BC membrane in solution with polymer materials or inorganic nanoparticles, impregnation and coating were explored to fabricate $\mathrm{BC}$ based composites with fascinating properties and wide range of application possibilities (Barud et al., 2011, 2015; Brown \& Laborie, 2007; Castro et al., 2014; Dal'Acqua et al., 2015; Gea et al., 2010; Gutierrez, Tercjak, et al., 2012; Gutierrez et al., 2013; Hebler \& Klemm, 2009; Jian et al., 2014; Li et al., 2009; Marins et al., 2011; Olsson et al., 2010; Quero et al., 2010; Tercjak et al., 2015). On the other hand, low molecular weight nematic liquid crystals (LC) are organic molecules with interesting properties especially a large optic and dielectric anisotropy (Doane, Golemme, West, Whitehead, \& Wu, 1988; Drzaic, 1988; Zhou, Collard, Park, \& Srinivasarao, 2002). Consequently, LC are one of the most convenient materials to control light by changes in the alignment of their molecules with external stimuli such as an electrical field, a temperature gradient and light (Campbell, Tasinkevych, \& Smalyukh, 2014; Doane et al., 1988; Drzaic, 1988; Ganesan, Wirges, Mellinger, \& Gerhard, 2010; Tercjak, Gutierrez, \& Mondragon, 2011; Tercjak, Gutierrez, Ocando, Peponi, \& Mondragon, 2009; Tercjak, Serrano, \& Mondragon, 2006; Zhou et al., 2002). For this reason, LC have been extensively combined with polymers to design novel materials for application in the field of thermo- and electro-optical devices, such as optical shutters, smart windows, optical sensors, memories and flexible displays (Doane, Vaz, Wu, \& Zumer, 1986; Herod \& Duran, 1998; Sumana \& Raina, 2005). However, based on our knowledge low molecular weight nematic liquid crystals have never been used in combination with BC.

Taken above into account, in present work three low molecular weight liquid crystals, 4'-(hexyloxy)-4-biphenyl carbonitrile (HOBC), 4'-(hexyl)-4-biphenyl-carbonitrile (HBC) and multicomponent nematic mixture E7, were used for the preparation of LC coated BC films. UV-vis spectroscopy was used to study optical properties and transparency of the fabricated HOBC, HBC and E7 coated BC films. Structural properties and the dispersion of the liquid crystals on the surface of the BC film were studied employing atomic force microscopy and optical microscopy. Moreover, transversal cross-section of the coating was investigated to check profundity of the liquid crystals impregnation through threedimensional BC nanofibers network. Photoluminescence properties of investigated liquid crystals coated BC films as a function of temperature were studied using spectrophotometer. Additionally, taken into account that nematic liquid crystals can have different respond to an applied voltage, tunneling atomic force microscopy measurement was employed to prove the effect of external stimuli on the liquid crystals coated BC films. Finally, mechanical properties were also studied using a material testing system (MTS).

\section{Experiment}

\subsection{Materials}

Two low molecular weight nematic liquid crystals used were 4'-(hexyloxy)-4-biphenyl carbonitrile (HOBC) and 4'-(hexyl)-4biphenyl-carbonitrile (HBC) supplied by Sigma-Aldrich, and used without further purification. Additionally, low molecular weight nematic liquid crystal E7 supplied by Merck was also used without further purification. The thermal transitions of used LCs are shown in Table 1 . These transitions were determinate by differential scanning calorimetry (DSC).

\subsection{Production of $B C$ inoculum}

The strain used was Gluconacetobacter xylinum (ATCC 23760) supplied by André Tosello Foundation, Campinas-SP, Brazil. It was cultured in Hestrim-Schramm medium (HS medium) that
Table 1

Crystal-nematic transition ( $\left.T_{\mathrm{C}-\mathrm{N}}\right)$ and nematic-isotropic transition $\left(T_{\mathrm{N}-\mathrm{I}}\right)$ of $\mathrm{HBC}$, HOBC and E7 as determined by DSC.

\begin{tabular}{lcl}
\hline Name & $T_{\mathrm{C}-\mathrm{N}}\left({ }^{\circ} \mathrm{C}\right)$ & $T_{\mathrm{N}-\mathrm{I}}\left({ }^{\circ} \mathrm{C}\right)$ \\
\hline HBC & 26 & 34 \\
HOBC & 59 & 76 \\
E7 & -10 & 58 \\
\hline
\end{tabular}

contained D-glucose glycose, yeast extract, peptone, di-sodium hydrogen phosphate $\left(\mathrm{Na}_{2} \mathrm{HPO}_{4}\right)$, citric acid, agar and purified water. Analytical grade chemicals were used as received. Before the bacterial strain inoculation the strain culture medium was sterilized and then was cultivated during 1 day at $28^{\circ} \mathrm{C}$ in an air circulating oven as conditioning chamber.

\subsection{Biosynthesis of bacterial cellulose}

BC culture medium was prepared according to the method previously reported by us (De Salvi et al., 2014; Tercjak et al., 2015). $45 \mathrm{~mL}$ of culture medium and $5 \mathrm{~mL}$ of the inoculum was cultivated during 3 days in static conditions at $28^{\circ} \mathrm{C}$ in a $250 \mathrm{~mL}$ Erlenmeyer flask in an air circulating oven. After this time, jelly-like BC pellicle was harvested and purified to eliminate the culture medium (to remove the cells and other impurities). The purification protocol was performed following the pathway published by Tercjak et al. (2015). Finally, purified BC pellicle was dried in order to obtain BC film.

\section{4. $L C / B C$ film preparation protocol}

Considering practical applications of designed materials, we proposed an easy method which can also be applied over largescale areas at ambient conditions relatively inexpensively. Thus, we fabricated $\mathrm{LC} / \mathrm{BC}$ films by a simple painting process. The BC film was used as support (substrate, hold) for the LC coating. BC film substrates were painted with LC solution ( $3 \mathrm{wt} \%$ in chloroform) in order to get LC coated BC film. To ensure the same quantity of the LC used for painting, $1 \mathrm{~mL}$ of the LC solution was employed for preparation of each sample. Prepared LC/BC films were dried in a vacuum oven at $30^{\circ} \mathrm{C}$ during $24 \mathrm{~h}$. Additionally, elemental analysis was realized to determine LC content in LC/BC films. Table 2 shows the carbon $(\mathrm{C})$, hydrogen $(\mathrm{H})$ and nitrogen $(\mathrm{N})$ contents of investigated materials.

\subsection{Characterization techniques}

Elemental Analysis of LC coated BC films was carried out using a Euro EA Elemental Analyzer made by EuroVector. Independent measurements were performed to measure $\mathrm{C}, \mathrm{H}$ and $\mathrm{N}$ contents.

The optical properties of BC film and nematic liquid crystal coated BC films were studied using a UV-3600, Shimadzu UV-VISNIR spectrophotometer in the wavelength range of $200-800 \mathrm{~nm}$. The average thickness of the samples used for this measurement was $20 \pm 3 \mu \mathrm{m}$.

Fourier transform infrared spectra (FTIR) were carried out in a Nicolet Nexus Spectra equipped with a Golden Gate single reflection diamond ATR accessory and were taken with a $2 \mathrm{~cm}^{-1}$ resolution in a wavenumber range from 4000 to $400 \mathrm{~cm}^{-1}$.

Table 2

Elemental analysis data of LC coated BC films.

\begin{tabular}{llll}
\hline Sample & C\% & H\% & N\% \\
\hline HOBC/BC & 46.53 & 6.40 & 0.5 \\
HBC/BC & 45.26 & 6.42 & 0.3 \\
E7/BC & 45.89 & 6.34 & 0.28 \\
\hline
\end{tabular}


Thermogravimetric analysis (TGA) was performed using a TGA/SDTA-851e equipment under air atmosphere at a heating rate

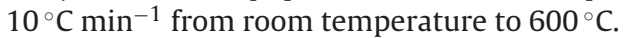

X-ray diffraction (XRD) was carried out on a Philips PW 1710 diffractometer. The $\mathrm{Cu} \mathrm{K} \alpha \mathrm{X}$-ray source was set to $40 \mathrm{kV}$ and $100 \mathrm{~mA}$ and the samples were examined over the angular range of $10-30^{\circ}$.

The surface homogeneity of investigated BC film and LC coated BC films at the macroscale was investigated using an optical microscope (OM) Nikon Eclipse E600. The investigated materials were analyzed in reflection mode and crossed polarizers. Micrographs were captured with a Color View 12 camera and analyzed using the AnalySIS Auto 3.2 software (Soft Imaging System $\mathrm{GmbH}$ ).

Morphological study of designed materials was performed using an atomic force microscopy (AFM). Corresponding AFM images were obtained by operating in a tapping mode with a scanning probe microscope Dimension ICON from Bruker equipped with an integrated silicon tip/cantilever having a resonance frequency of $300 \mathrm{kHz}$. Scan rates ranged from 0.7 to $1.2 \mathrm{~Hz} \mathrm{~s}^{-1}$. In order to obtain repeatable results, different regions of the specimens were scanned to choose representative AFM images. Taking into account that obtained height and phase AFM images were very similar, only AFM phase images are shown.

Scanning electron microscopy (SEM) images transverse crosssection of BC film and LC coated BC films were obtained using a field emission scanning electron microscope JEOL JSM-7500F. All investigated films were sputter coated by a carbon layer before SEM measurement.

Mechanical properties at the macroscale level of BC film and designed LC/BC films were investigated at room temperature on a tensile testing machine MTS Insight 10 equipment with load cell of $250 \mathrm{~N}$. Specimens of $20 \mathrm{~mm}$ length and $5 \mathrm{~mm}$ width were tested with strain rate of $1 \mathrm{~mm} / \mathrm{min}$. All samples were conditioned for $48 \mathrm{~h}$ under vacuum prior to testing. Elongation at break and tensile strength were calculated as an averaged out from five test specimen data.

The photoluminescence properties of the investigated LC coated BC films were determined using a Felix32 spectrophotometer of Photon Technology International (PTI) equipped with a temperature controller. Fluorescence emissions spectra for all investigated materials were recorded during the heating/cooling cycles from 10 to $50^{\circ} \mathrm{C}$ at a rate of $5{ }^{\circ} \mathrm{C} / \mathrm{min}$. The excitation wavelengths were $330 \mathrm{~nm}$ for HOBC, $311 \mathrm{~nm}$ for $\mathrm{HBC}$ and $342 \mathrm{~nm}$ for E7. Measurements were repeated for several times to confirm the ability of designed $\mathrm{BC}$ based films to act as photoluminescence switchable materials.

Conductive properties of the investigated materials were measured using tunneling atomic force microscopy (TUNA). The measurements were carried out using PeakForce TUNA (PFTUNA) mode under ambient conditions. The PF-TUNA probe was equipped with the $\mathrm{Pt} / \mathrm{Ir}$ coating tip. The sample dimensions $(10 \mathrm{~mm} \times 3 \mathrm{~mm} \times 0.005 \mathrm{~mm})$ were always the same in order to avoid confusion in results interpretation since the response of the samples is proportional to the applied voltage and inversely proportional to the thickness (Gutierrez, Tercjak, \& Mondragon, 2010; Tercjak, Gutierrez, Ocando, \& Mondragon, 2010).

\section{Results and discussion}

UV-vis spectra of BC film and LC coated BC films are shown in Fig. 1. Additionally, UV-vis spectra of pristine LCs are shown in Fig. S1. The top left inset shows visual transparency of all investigated films. Digital images of transparency clearly visualized high transparency of $\mathrm{HBC}$ and E7 coated BC films. On the contrary, the transparency of $\mathrm{HOBC}$ coated BC film is lower than the one observed for the BC film.

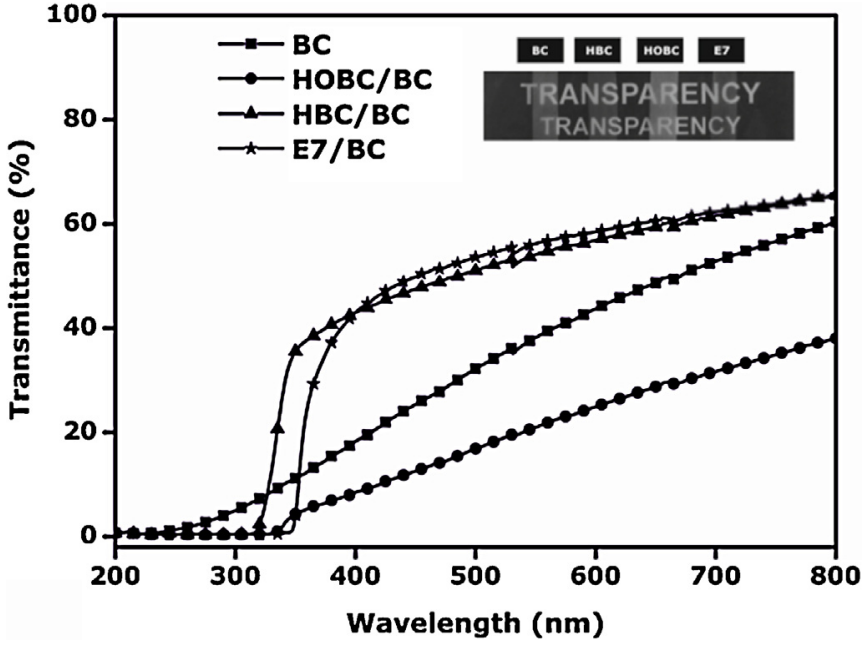

Fig. 1. UV-vis transmittance spectra of $B C$ film and $L C$ coated $B C$ films. The inset shows a digital image of investigated films.

The transparency of investigated LC coated BC films was confirmed by their UV-vis transmittance spectra at visible wavelength range. The transmittance of $\mathrm{HBC}$ and $\mathrm{E} 7$ coated $\mathrm{BC}$ films was higher than BC film, being $62 \%$ for both LC coated films and $52 \%$ for BC film at wavelength equal to $700 \mathrm{~nm}$. Under the same measurement conditions, the HOBC coated BC film had the lowest transmittance being $30 \%$ at wavelength equal to $700 \mathrm{~nm}$. Here it should be pointed out that the transparency of the LC coated BC films depends strongly on the state of each nematic liquid crystal at room temperature. As it is well known, at room temperature HBC and E7 liquid crystals are in liquid state since they possess their nematic-isotropic $\left(T_{\mathrm{N}}-T_{\mathrm{I}}\right)$ transition at temperature lower than room temperature (Table 1). On the contrary, HOBC liquid crystal is in solid state.

According to the transmittance in the UV light range, all LC coated BC films exhibited almost perfect UVC and UVB light shielding efficiency. As shown in Fig. 1, transmittance values in this region were close to zero. This behavior suggests that the LC coating shield UVC and UVB that can decrease UV irradiation damage.

As can be easily observed, the E7/BC film showed the highest UV-shielding efficiency up to $348 \mathrm{~nm}$. In the case of HBC and HOBC the transmittance was equal to zero up to wavelength of 335 and $320 \mathrm{~nm}$, respectively. Here it should be pointed out that taken into account the UV-vis spectra of pristine LCs (Fig. 1S) one can conclude that HOBC, HBC and E7 liquid crystal transferred UV-shielding efficiency to LC coated BC films.

Taking into account both low absorption in the visible light range and UV light absorption, E7/BC film had optimal properties, hence high UV-shielding efficiency and high visible light transparency.

ATR-FTIR spectra of BC film and LC coated BC films are shown in Fig. 2.

ATR-FTIR spectrum of BC film showed characteristics bands of bacterial cellulose at $3345 \mathrm{~cm}^{-1}(\mathrm{O}-\mathrm{H}$ stretching of cellulose type I), $3240 \mathrm{~cm}^{-1}$ (hydrogen bonded $\mathrm{O}-\mathrm{H}$ ), $2895 \mathrm{~cm}^{-1}$ ( $\mathrm{CH}$ stretching of $\mathrm{CH}_{2}$ groups), $2854 \mathrm{~cm}^{-1}$ ( $\mathrm{CH}_{2}$ asymmetric stretching), $1650 \mathrm{~cm}^{-1}$ (bending motion of absorbed water $\mathrm{H}-\mathrm{O}-\mathrm{H}), 1426 \mathrm{~cm}^{-1}\left(\mathrm{CH}_{2}\right.$ symmetric bending), $1365 \mathrm{~cm}^{-1}$ ( $\mathrm{CH}$ bending), $1160 \mathrm{~cm}^{-1}$ (C-O-C asymmetric stretching), and $1060 \mathrm{~cm}^{-1}$ (C-O stretching) (Barud et al., 2008; Czaja, Romanowicz, \& Brown, 2004). As expected, ATRFTIR spectra of HOBC, HBC and E7 coated BC films were similar to ATR-FTIR spectrum of BC film. Under the same measurement conditions, the intensity of the - $\mathrm{OH}$ stretching bands was slightly higher for LC coated BC films however any changes in wavenumber was 


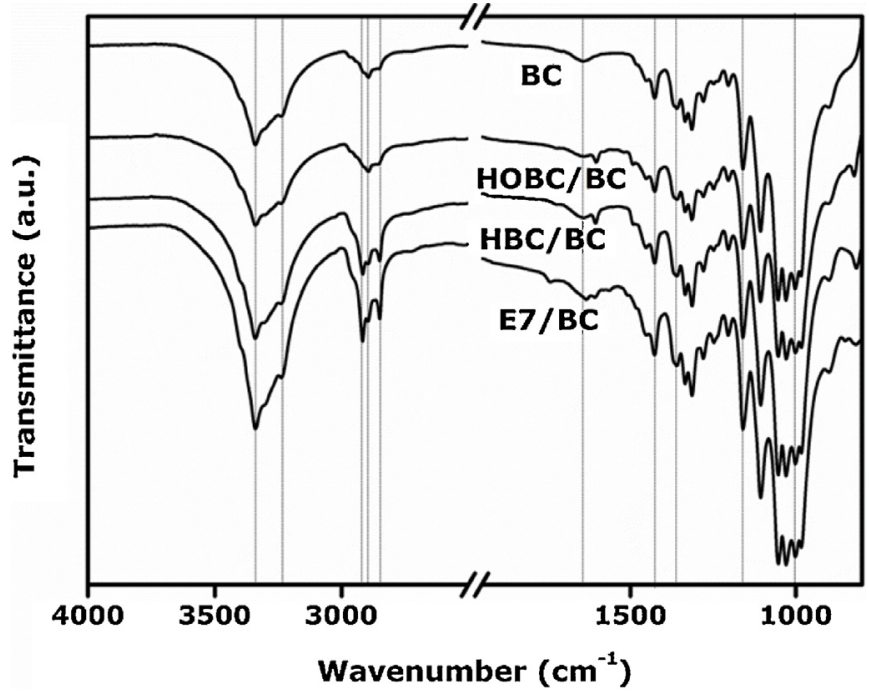

Fig. 2. ATR-FTIR spectra of BC film and LC coated BC films.

detected. Simultaneously, the intensity of the $\mathrm{C}-\mathrm{H}$ stretching vibration of $\mathrm{CH}_{2}$ and $\mathrm{CH}_{3}$ groups was higher for $\mathrm{HBC}$ and $\mathrm{E} 7$ coated $\mathrm{BC}$ films and in both cases peak at $2915 \mathrm{~cm}^{-1}$ was also clearly detected in the $\mathrm{CH}_{2}$ and $\mathrm{CH}_{3}$ stretching region. These peaks can be attributed to the stretching vibration of $\mathrm{CH}_{2}$ group present in LC. The difference between ATR-FTIR spectra of HBC and E7 coated BC films if compare with ATR-FTIR spectrum of HOBC coated BC film can be related to the fact that at room temperature $\mathrm{HBC}$ and $\mathrm{E7}$ liquid crystals are in liquid state whereas HOBC liquid crystal is in solid state. Furthermore, ATR-FTIR spectra of BC film and LC coated BC films do not show the formation of any additional bands if compare with ATR-FTIR spectra of neat BC film, which confirm lack of chemical bonds between HOBC, HBC or E7 and bacterial cellulose.

As shown in Fig. 3, the thermal stability of the BC film was not affected by the LC coating. Thermogravimetric curves of $\mathrm{HOBC} / \mathrm{BC}$, $\mathrm{HBC} / \mathrm{BC}$ and $\mathrm{E} 7 / \mathrm{BC}$ films are almost the same as thermogravimetric curve of $B C$ film (see Fig. 3a). Thus, all investigated $B C$ films followed the same decomposition process consisted of three steps at around 100,350 and $490^{\circ} \mathrm{C}$. The first minor weight loss from ambient to $100^{\circ} \mathrm{C}$ was attributed to $\mathrm{BC}$ films dehydration (Li et al., 2009) whereas other two weight losses corresponded to the thermal degradation of the BC films (Barud et al., 2008). These three steps decomposition process was clearly visualized as peaks in Fig. 3b correspond to derivative weight loss of each investigated BC films. Deeper analysis of the last decomposition steps of all BC films at around $480-490^{\circ} \mathrm{C}$ indicated slight difference between LC coatings. As expected, taken into account organic character of $\mathrm{BC}$ and LC complete degradation of investigated coatings took place for the temperature higher than $490^{\circ} \mathrm{C}$.

The semicrystalline character of BC film and LC coated BC films were studied using X-ray diffraction. The X-ray diffractograms of BC film, and HOBC, HBC and E7 coated BC films are shown in Fig. 4.

XRD pattern of BC film showed three peaks at $2 \Theta$ of approximately $14.6^{\circ}, 17.1^{\circ}$ and $22.9^{\circ}$, which correspond to the primary diffraction of the (101), (1 0101$)$ and (0 0 2) polymorphism cellulose I (Pircher et al., 2014; Yu, Qin, Yan, \& Yao, 2014). LC coated BC films revealed $X$-ray diffractograms similar to this of $B C$ film with the maximum of the peaks detected almost at the same $2 \Theta$ values.

In order to study the effect of the LC coatings on the morphology of the BC film, AFM measurements were performed. As shown in Fig. 5 AFM phase images of BC film and HOBC, HBC and E7 coated $B C$ films showed highly interconnected three-dimensional network consists of nanofibers characteristic for BC film. As visualized in
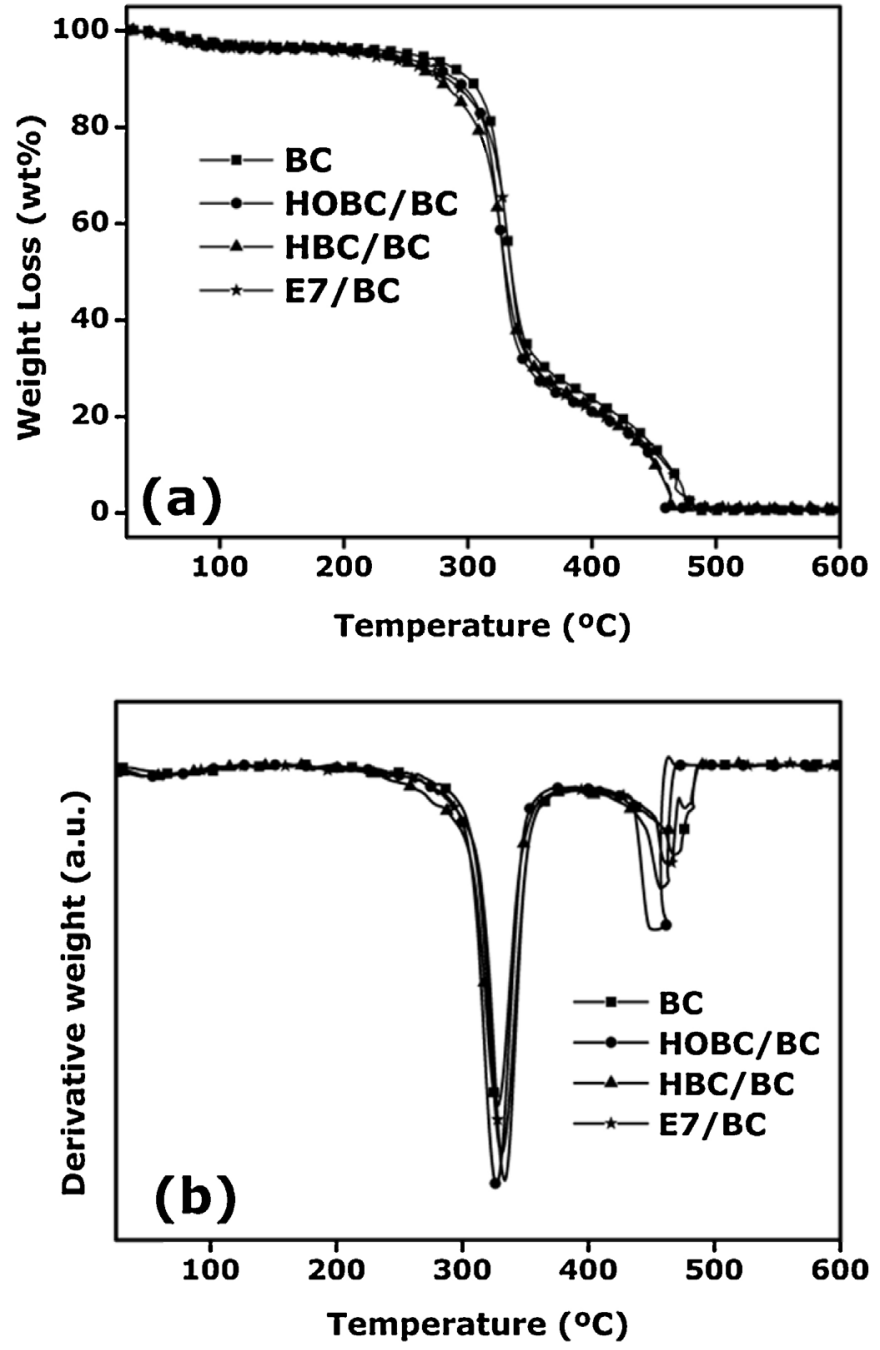

Fig. 3. (a) Thermogravimetric and (b) differential thermogravimetric curves of $B C$ film and LC coated BC films.

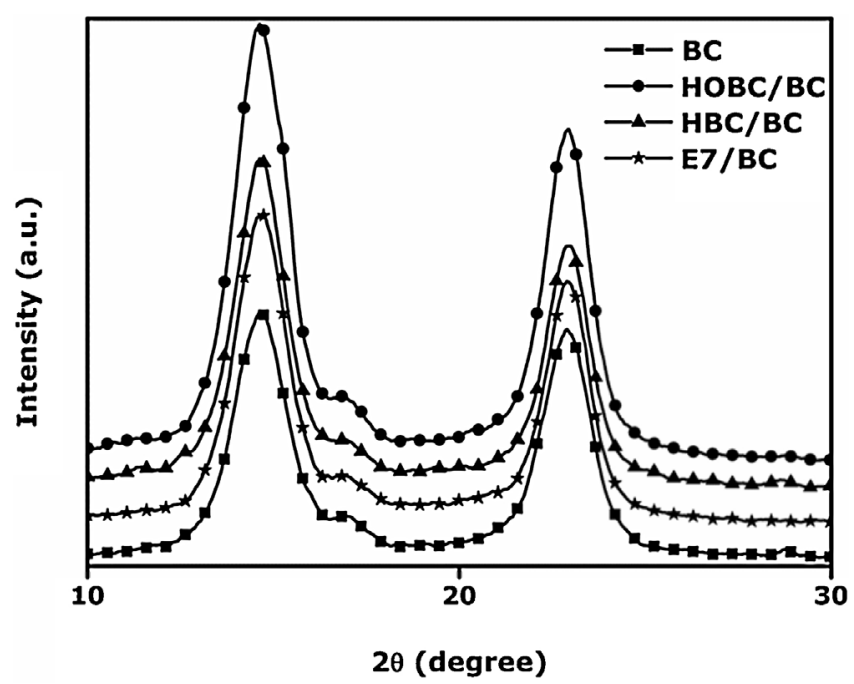

Fig. 4. X-ray diffraction of BC film and LC coated BC films. 


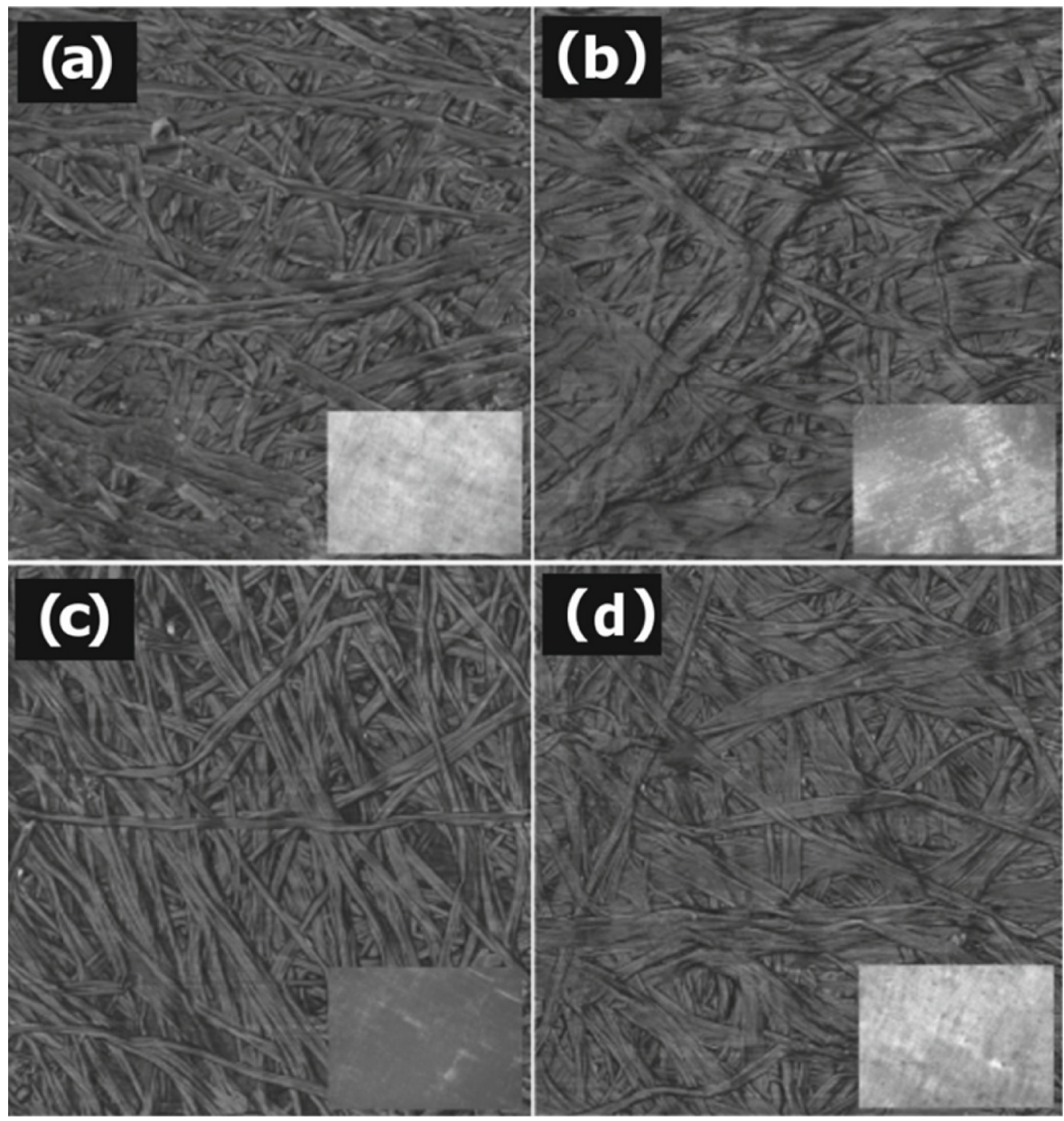

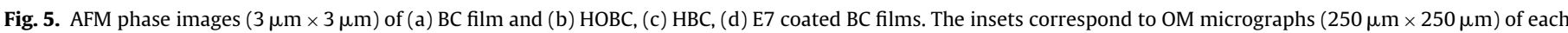
BC film taken between crossed polarizers.

Fig. 5a more than $3 \mu \mathrm{m}$ longer nanofibers with a diameter of around 80-190 nm consist of 2-4 fibrils, which twisted together forming braids as can be clearly observed on the investigated surface of the BC film.

On the contrary to the BC film, as show in the AFM phase image of the HOBC coated BC film (Fig. 5b), liquid crystal covers the surface of the nanofibers. Consequently, nanometric size fibrils are not distinguished in the case of $\mathrm{HOBC}$ coated BC film. AFM phase image of $\mathrm{HBC}$ coated BC film (Fig. 5c) showed that the surface structure of BC film covered with $\mathrm{HBC}$ possessed similar surface properties as BC film. In this case similarly to $\mathrm{BC}$ film, the fibril structure of the nanofibers on the surface of HBC coated BC film was also clearly observed. AFM phase image of E7 coated BC film (Fig. 5d) indicates that the E7 liquid crystal act on a similar way as HOBC liquid crystal. Thus, once more for many nanofibers visualized in E7 coated BC film the fibril structure of nanofibers could not be detected. Therefore the E7 liquid crystal covered partially the surface of nanofibers of BC. Additionally, it should be pointed out that OM micrographs of each investigated samples (Fig. 5, bottom insets) confirmed homogenous distribution of the each nematic liquid crystals on the surface of the BC film at the macroscopic scale.

To better understand the different effects of LC coating on the BC nanofibers one should take into account that as mentioned above at room temperature $\mathrm{HBC}$ and E7 liquid crystals are in liquid state whereas HOBC liquid crystal is in solid state.

In order to study the penetration of nematic liquid crystals through the three-dimensional nanofibers network of the BC film, the cross-section surface of fabricated uncoated and LC coated BC films were investigated employing SEM technique. As shown in Fig. 6a I, the cross-section of the BC film shows typical multi-layered structure of nanofibers network with the thickness of around $5 \mu \mathrm{m}$. Here it should be pointed out that the thickness of the bacterial cellulose depends strongly on the cultivation time and preparation protocol (Barud et al., 2015; Brown \& Laborie, 2007; Tercjak et al., 2015). SEM image of the cross-section of HOBC coated BC film (Fig. 6b I) proved that HOBC liquid crystal penetrate throughout multi-layered structure of nanofibers network of BC film changing completely their cross-section. As clearly observed in Fig. 6b II, HOBC liquid crystals form homogenous coating not only at the surface of the $\mathrm{BC}$ film but also through three-dimensional nanofibers structure of the BC film (compare Fig. 6a II and b II). The thickness of HOBC coated BC film increase from $\sim 5 \mu \mathrm{m}$ for BC (Fig. 6a I) film to around $9 \mu \mathrm{m}$ (Fig. $6 \mathrm{~b} \mathrm{I}$ ). Taken into account the same fabrication conditions for all investigated $\mathrm{BC}$ films this thickness increase can be related to the swelling of the $\mathrm{BC}$ film as effect of penetration of the HOBC liquid crystals through multi-layered structure of nanofibers network of $\mathrm{BC}$ film. The cross-sections of HBC and E7 coated BC films (Fig. 6c I and d I) are different, compared with the one observed for HOBC coated BC film. Similarly to the BC film, the multi-layered structure of nanofibers network was clearly visualized (Fig. 6c II and d II). However, the thickness of these LC coated $\mathrm{BC}$ films is almost two times larger, being $\sim 8 \mu \mathrm{m}$ and $\sim 9 \mu \mathrm{m}$ for HBC and E7 coated BC films, respectively. As visualized in Fig. 6a-d $\mathrm{I}$, this thickness increase once more indicates that LCs penetrate throughout the multi-layered structure of BC film. As visualized in the cross-section SEM images, the difference between HOBC coated 

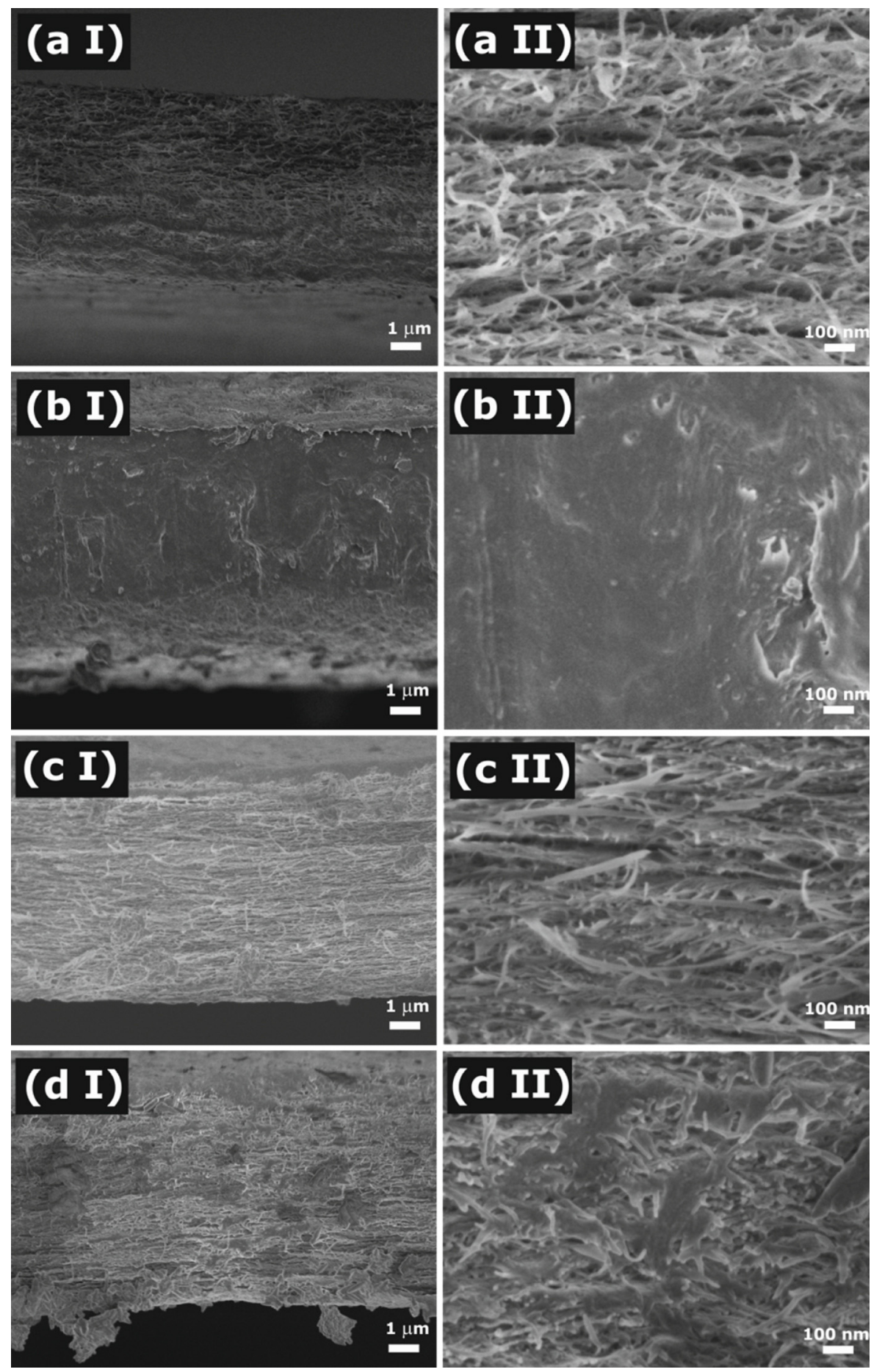

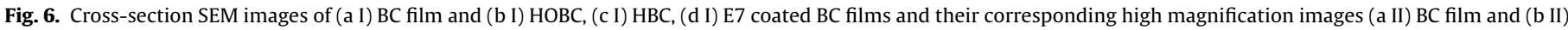
HOBC, (c II) HBC, (d II) E7 coated BC films.

BC film and the others two, HBC and E7 coated BC films, can be related to the fact that, as mentioned above, at room temperature HOBC liquid crystal is in solid state whereas HBC and E7 liquid crystals are in liquid state. Here, it should be pointed out that the SEM results are in good agreement with AFM results.

Fig. 7 shows elongation at break (\%) and tensile modulus (E) results. Concerning elongation at break values, no significant difference was achieved for BC film, НOBC and HBC coated BC films. However for E7 coated BC film the value slightly increased, being $\sim 3.2 \%$. It is worth to note that this slight increase means that the elongation at break for E7 coated BC film is two times higher than for BC film, which can be related to higher plasticization effect of E7 compared to HBC and HOBC.

On the other hand (Fig. 7b), tensile modulus (E) values slightly decreased for LC coated BC films compared to $\mathrm{E}$ of the BC film. BC film possessed the highest tensile modulus whereas $\mathrm{HBC}$ coated BC film had the lowest one, being 12.4 and 8.6 GPa, respectively. The decrease in the overall tensile modulus of LC coated BC films could be attributed to the penetration of LC through BC threedimensional nanofibers network. The interfibril interactions of $\mathrm{BC}$ may be weakened by reduced hydrogen bonding density due to the addition of hydrophobic LC. Taking into account the large error 
a

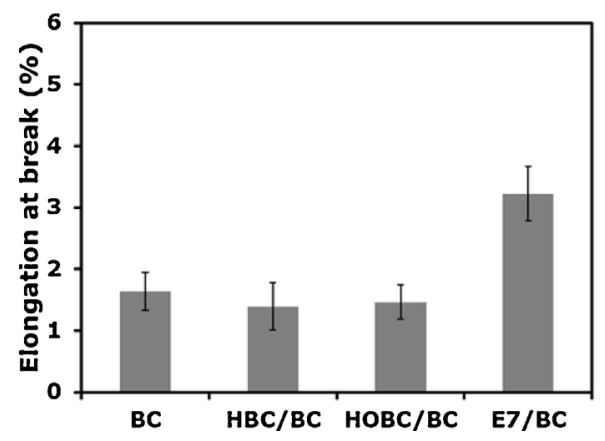

b

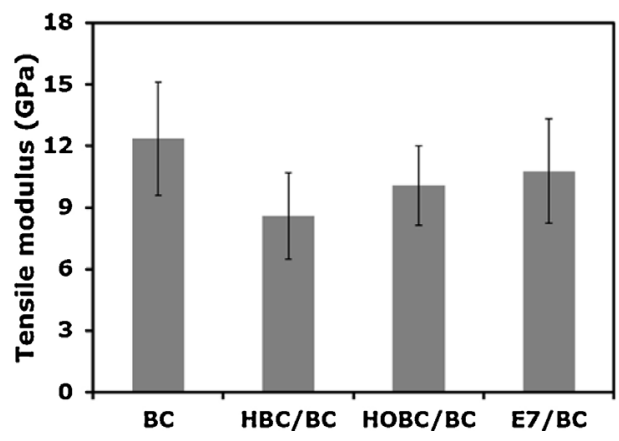

Fig. 7. (a) Elongation at break and (b) tensile modulus of BC film and LC coated BC film.

bars, tensile modulus values obtained for the three LC coated BC films were very similar. In fact, large error bars hindered a deeper analysis of obtained results.

In order to verify the photoluminescence properties of designed films, the fluorescence emission spectra of each film coated with different liquid crystals as a function of the temperature were carried out. Fig. 8 shows the fluorescence emission spectra of $B C$ films impregnated with HOBC, HBC and E7. HOBC coated BC film possessed well-defined emission peak at around $372 \mathrm{~nm}$ at $50^{\circ} \mathrm{C}$, which is almost not detected at $10^{\circ} \mathrm{C}$, in which the $\mathrm{HOBC}$ liquid crystal was in crystalline state. Here it should be pointed out that fluorescence emission spectra were taken as a function of temperature during heating/cooling allowing to visualise well-defined, reversible increase/decrease of emission spectrum of HOBC coated
BC film at $50^{\circ} \mathrm{C}$ and at $10^{\circ} \mathrm{C}$. This process was reversible in more than ten heating/cooling cycles, which was strongly related to nematic liquid crystals properties able to change in the alignment of their molecules in function of temperature (ON/OFF liquid crystal states) (Tercjak et al., 2006, 2009, 2010).

In the same way HBC coated BC film revealed the emission peak at $363 \mathrm{~nm}$ at $50^{\circ} \mathrm{C}$ and strong, more than ten times, reversible decreased of the emission peak at $10^{\circ} \mathrm{C}$. This emission spectrum taken during heating/cooling showed intensity almost three times lower at $10^{\circ} \mathrm{C}$ than at $50^{\circ} \mathrm{C}$ confirming capability of this $\mathrm{BC}$ based film to photoluminescence switching as a function of temperature. Here should be pointed out that similarly to HOBC coated BC film, the photoluminescence switching of $\mathrm{HBC}$ coated $\mathrm{BC}$ film took place almost at the same temperature during heating/cooling process.
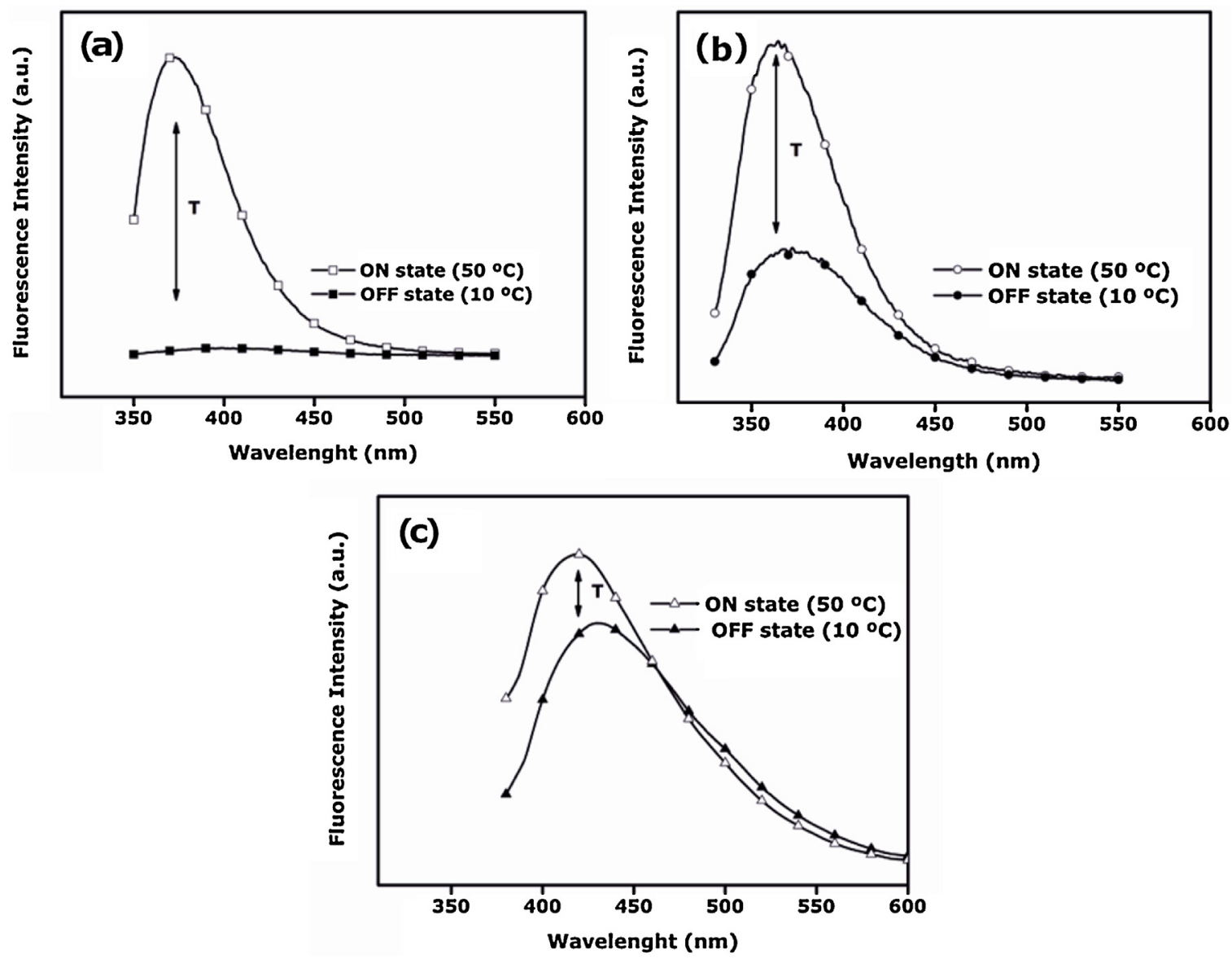

Fig. 8. Fluorescence emission spectra of (a) HOBC, (b) HBC, (c) E7 coated BC films. 


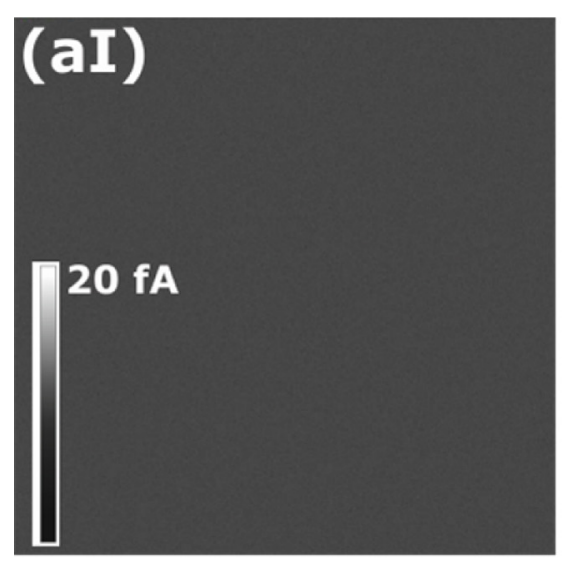

\section{(aII)}
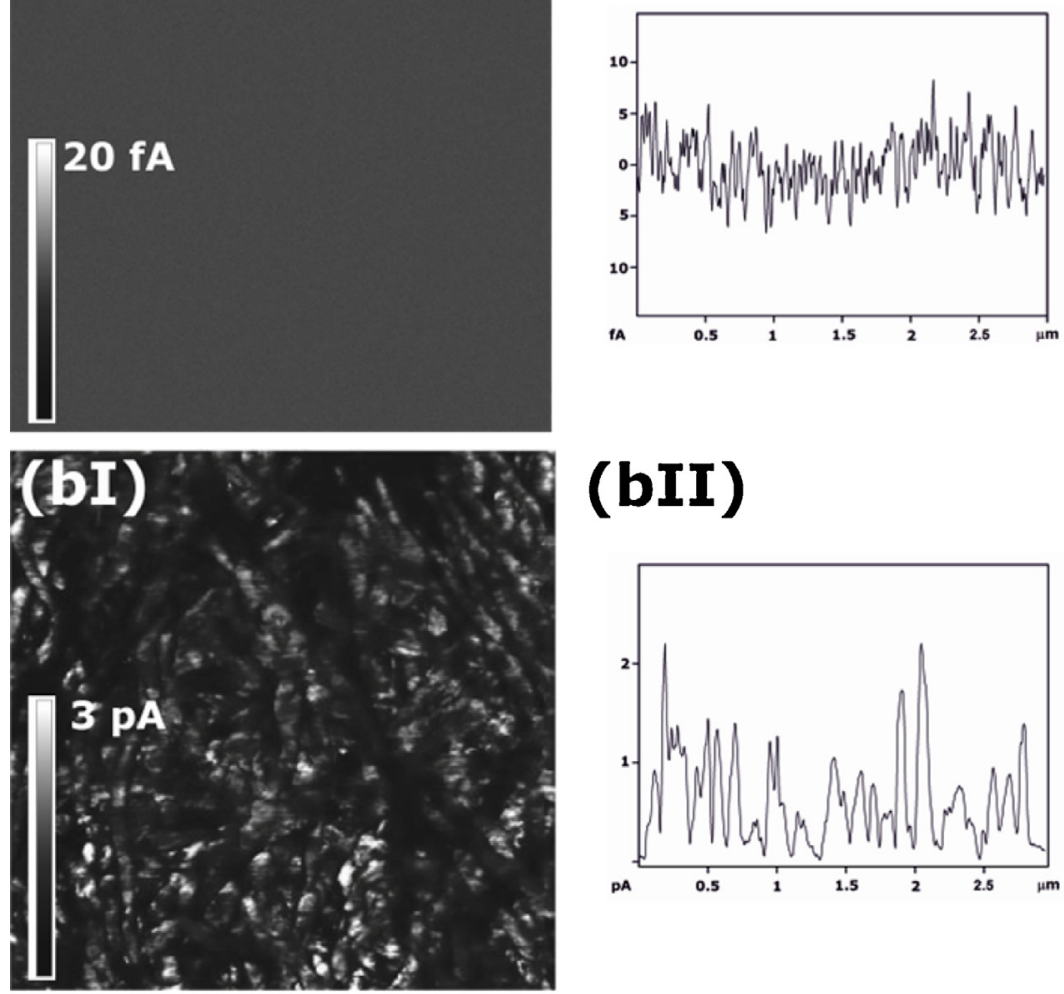

\section{(bII)}
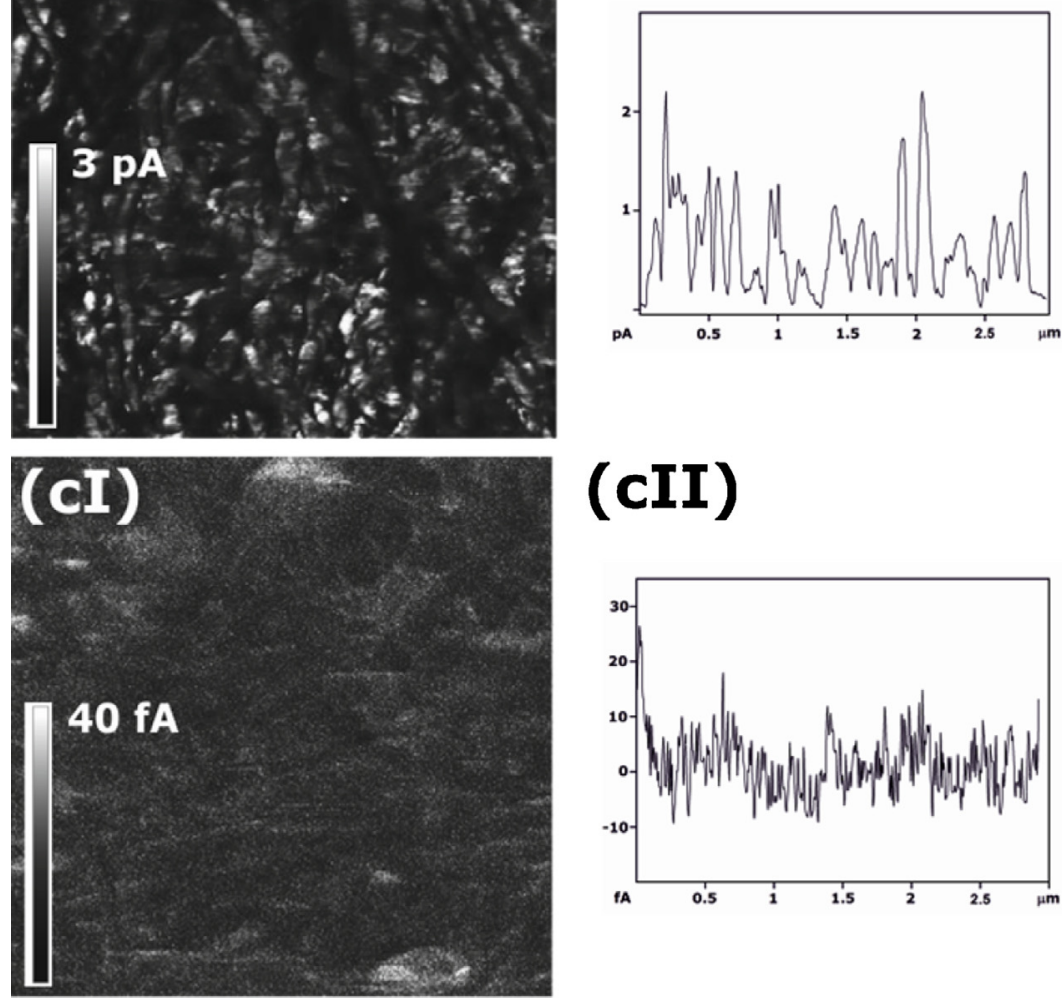

\section{(cII)}
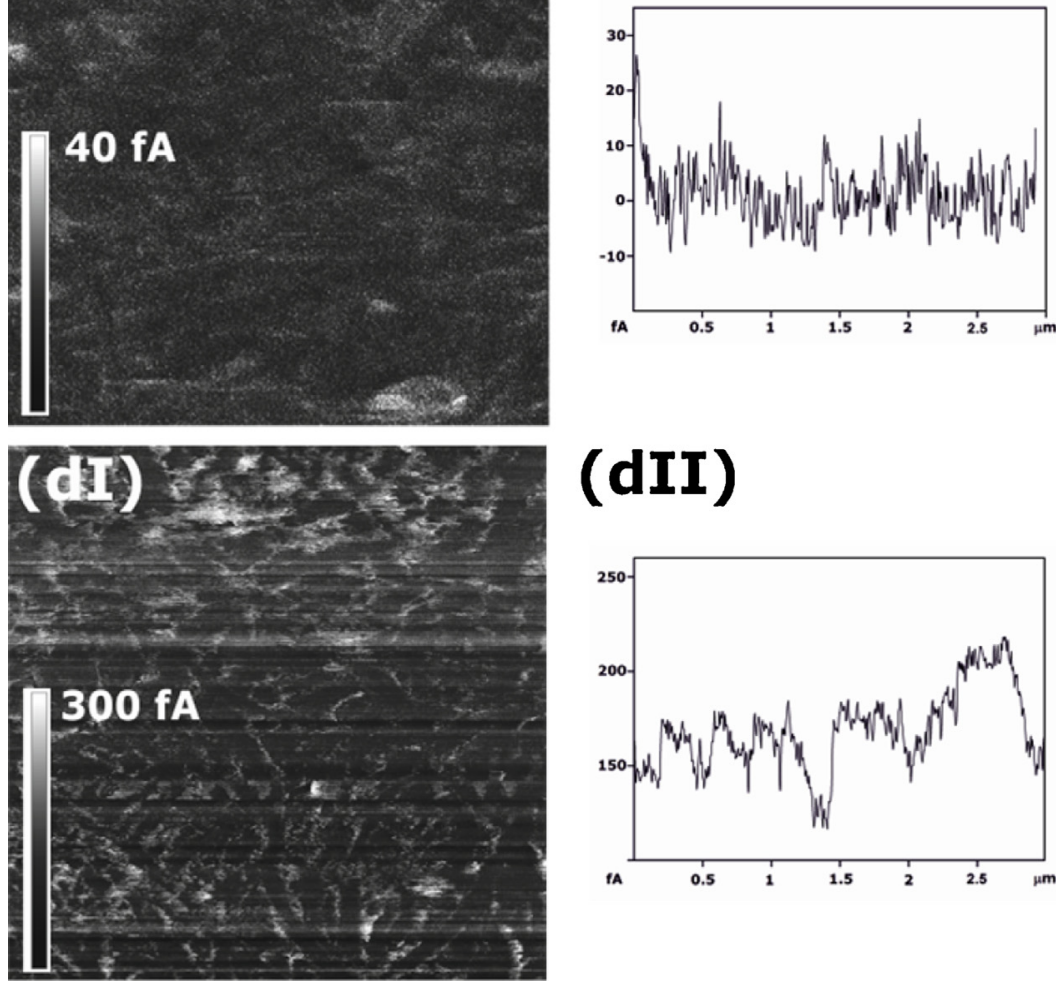

\section{(dII)}

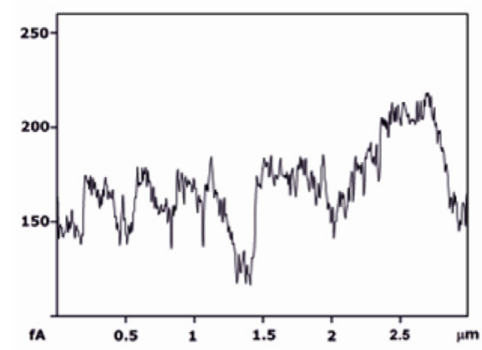

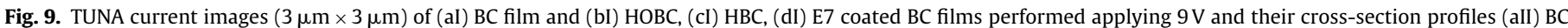
film and (bII) HOBC, (cII) HBC, (dII) E7 coated BC films. 
The impregnation of BC film with E7 liquid crystal presented emission spectra at the highest wavelength being $419 \mathrm{~nm}$ at $50^{\circ} \mathrm{C}$. Furthermore, at $10^{\circ} \mathrm{C}$ the fluorescence intensity of the emission spectra became much lower than at $50^{\circ} \mathrm{C}$ being thermoresponsive in more than ten heating/cooling cycles. Here, it should be pointed out that the smallest intensity changes in fluorescence emission during photoswitching for E7 coated BC film if compare with changes during photoswitching for $\mathrm{HOBC}$ and $\mathrm{HBC}$ coated $\mathrm{BC}$ films is related with the fact that E7 coated $\mathrm{BC}$ film needs lower temperature to be in solid state.

Fig. S2 shows fluorescence emission spectra as a function of temperature for employed pristine LCs. Obtained results verified that, under the same measurement conditions, HOBC, HBC and E7 possess switchable photoluminescence properties. Thus, LC coated BC films exhibit novel properties due to cooperative effects between LC and BC, especially, taken into that the LC coated BC films show emission in different range if compare to corresponding pristine LC.

Tunneling atomic force microscopy (TUNA) was successfully applied to investigate the charge distribution and conductivity level of LC coated BC films. This conductive behavior was expected since as is well known from the literature, nematic liquid crystals can change from OFF/ON state using external stimuli such as temperature, electric or magnetic field (Tercjak et al., 2006, 2011, 2010; Tercjak, García, \& Mondragon, 2008). Here, it should be pointed out that conductive response of the HOBC in polymer dispersed liquid crystals based on thermosetting systems nanostructured by poly(styrene-b-ethylene oxide) block copolymer was previously published by us elsewhere (Tercjak et al., 2010).

TUNA current images and corresponding cross-section profiles are shown in Fig. 9. TUNA current images were acquired applying $9 \mathrm{~V}$ between AFM chuck and AFM tip and the TUNA images represent the electric current passed between AFM tip and investigated BC films after applied voltage. Here, it should be mentioned that bright domains, in TUNA-current profiles, correspond to areas with higher conductivity variations, while the dark areas correspond to ultra-low current.

As expected, no bright domains appeared in TUNA image of BC film (Fig. 9aI), which indicates non-conductive behavior (Gutierrez et al., 2010; Tercjak et al., 2010) of this material. Thus, the response obtained for the BC film was observed at the noise level of the TUNA measurement as clearly visualized in a typical crosssection profile extracted from the TUNA current image (Fig. 9aII) (10 fA).

HOBC coated BC film shows higher conductivity values as easily distinguish in TUNA image and corresponding profile. Local higher conductivity peaks were detected along the analyzed sample surface. The intensity of these peaks, correspond to bright domains on the TUNA image. For HOBC coated BC film the highest current value obtained was $\sim 2$ pA. Moreover, additionally to high local current peaks, a constant current value was detected indicating that the current passed throughout the HOBC coated BC film varied homogeneously. Thus, taken into account that only $\mathrm{HOBC}$ can respond to the applied voltage it seems to be that the HOBC liquid crystal completely covered fibers of the $\mathrm{BC}$ film. In the case of $\mathrm{HBC}$ coated $\mathrm{BC}$ film, the current image was almost dark, which indicates poor conductive properties and conductivity value, as marked in the corresponding profile, was $\sim 20 \mathrm{fA}$. This small value was at the noise level of TUNA measurement. Under the same measurement conditions, E7 coated BC film led to higher conductivity values if compared with $\mathrm{HBC}$ coated $\mathrm{BC}$ film and consequently several bright conductive areas appeared in TUNA image. The corresponding profile indicates that the current value was nor higher than $250 \mathrm{fA}$ which correspond to a low conductivity level.

Therefore, under the same measurement and preparation conditions, HOBC and E7 coated BC films respond on the voltage applied to the AFM tip. The conductive response of HOBC coated $\mathrm{BC}$ film can be better understood if one can take into account that at room temperature HOBC liquid crystal is in solid state whereas HBC and E7 liquid crystals are in liquid state. Additionally, it is worth to note that as mentioned above all investigated LC coated BC films had the same dimensions for TUNA measurement since thickness has strong effect on conductive response.

\section{Conclusion}

Three low molecular weight liquid crystals $\mathrm{HOBC}$, HBC and E7 were used for impregnation of BC film. This easy way of preparation lead to highly transparent systems with UVC and UVB protection as confirm by UV-vis spectroscopy. Atomic force microscopy confirmed that LC coated films maintained interconnected three-dimensional network typical for BC film and, as confirmed by $\mathrm{OM}$, the whole surface was homogeneously covered by liquid crystals. The cross-section SEM images confirmed that HOBC, HBC and E7 liquid crystals not only covered surface of BC films however they were present also in between the layers of the three-dimensional network of BC films. Developed LC coated films maintained photoluminescence properties of the liquid crystals lead to thermoresponsive materials. Additionally, TUNA measurement confirms that depending on the solid or liquid state of the HOBC, HBC and E7 liquid crystal they can respond on the voltage applied to the AFM tip.

Finally, novel strategy to design bacterial cellulose based materials with thermoresponsive and conductive properties, which maintain mechanical properties and thermal stability of the BC film was developed.

\section{Acknowledgments}

This work was supported by Brazilian agencies CNPq, FAPESP and CAPES. J.G. acknowledges UPV/EHU for Postdoctoral Fellow (ESDOC13/059), A.T. acknowledges MICINN for the Ramon y Cajal program (RYC-2010-05592) and Brazilian agency FAPESP for Visiting Professor Fellow (REF: 2014/24692-1). Moreover, we are grateful to the Macrobehavior-Mesostructure-Nanotechnology and X-Ray SGIker units of the UPV/EHU.

\section{Appendix A. Supplementary data}

Supplementary data associated with this article can be found, in the online version, at doi:10.1016/j.carbpol.2016.02.019.

\section{References}

Abeer, M. M., Amin, M. C. I. M., \& Martin, C. (2014). A review of bacterial cellulose-based drug delivery systems: Their biochemistry, current approaches and future prospects. Journal of Pharmacy and Pharmacology, 66, 1047-1061.

Almeida, I. F., Pereira, T., Silva, N. H. C. S., Gomes, F. P., Silvestre, A. J. D., Freire, C. S. R., et al. (2014). Bacterial cellulose membranes as drug delivery systems: An in vivo skin compatibility study. European Journal of Pharmaceutics and Biopharmaceutics, 86, 332-336.

Barud, H. S., Barrios, C., Regiani, T., Marques, R. F. C., Verelst, M., Dexpert-Ghys, J. et al. (2008). Self-supported silver nanoparticles containing bacterial cellulose membranes. Materials Science and Engineering C, 28, 515-518.

Barud, H. S., Caiut, J. M. A., Dexpert-Ghys, J., Messaddeq, Y., \& Ribeiro, S. J. L. (2012) Transparent bacterial cellulose-boehmitecepoxi-siloxane nanocomposites. Composites, Part A, 43, 973-977.

Barud, H. S., Souza, J. L., Santos, D. B., Crespi, M. S., Ribeiro, C. A., Messaddeq, Y., et al. (2011). Bacterial cellulose/poly(3-hydroxybutyrate) composite membranes carbohydrate polymers. Carbohydrate Polymers, 83, 1279-1284.

Barud, H. S., Tercjak, A., Gutierrez, J., Viali, W. R., Nunes, E. S., Ribeiro, S. J. L., et al. (2015). Biocellulose-based flexible magnetic paper. Journal of Applied Physics 117(17), B734.

Brown, E. E., \& Laborie, M. P. G. (2007). Bioengineering bacterial cellulose/poly(ethylene oxide) nanocomposites. Biomacromolecules, 8 , 3074-3081. 
Bulota, M., Tanpichai, S., Hughes, M., \& -Eichhorn, S. J. (2012). Micromechanics of TEMPO-oxidized fibrillated cellulose composites. ACS Applied Materials \& Interfaces, 4, 331-337.

Campbell, M. G., Tasinkevych, M., \& Smalyukh, I. I. (2014). Topological polymer dispersed liquid crystals with bulk nematic defect lines pinned to handlebody surfaces. Physical Review Letters, 112, 197801.

Castro, C., Vesterinen, A., Zuluaga, R., Caro, G., Filpponen, I., Rojas, O. J., et al. (2014). In situ production of nanocomposites of poly(vinyl alcohol) and cellulose nanofibrils from Gluconacetobacter bacteria, effect of chemical crosslinking. Cellulose, 21, 1745-1756.

Czaja, W., Romanowicz, D., \& Brown, R. M. (2004). Structural investigations of microbial cellulose produced in stationary and agitated culture. Cellulose, 11, 403-411.

Dal'Acqua, N., de Mattos, A. B., Krindges, I., Pereira, M. B., Barud, H. S., Ribeiro, S. J. L., et al. (2015). Characterization and application of nanostructured films containing $\mathrm{Au}$ and $\mathrm{TiO}_{2}$ nanoparticles supported in bacterial cellulose. Journal of Physical Chemistry C, 119, 340-349.

De Salvi, D. T. B., Barud, H. S., Treu-Filho, O., Pawlicka, A., Mattos, R. I., Raphael, E., et al. (2014). Preparation, thermal characterization, and DFT study of the bacterial cellulose triethanolamine system. Journal of Thermal Analysis and Calorimetry, 118, 205-215.

Doane, J. W., Golemme, A., West, J. L., Whitehead, J. B., \& Wu, B. G. (1988). Polyme dispersed liquid crystals for display application. Molecular Crystals and Liquid Crystals, 165, 511-532.

Doane, J. W., Vaz, N. A., Wu, B. G., \& Zumer, S. (1986). Field controlled light scattering from nematic microdroplets. Applied Physics Letters, 48, 269-271.

Drzaic, P. S. (1988). Reorientation dynamics of polymer dispersed nematic liquid crystal films. Liquid Crystals, 11, 1543-1559.

Fu, L., Zhang, J., \& Yang, G. (2013). Present status and applications of bacterial cellulose-based materials for skin tissue repair. Carbohydrate Polymers, 92, 1432-1442.

Galland, S., Andersson, R. L., Salajkova, M., Strom, V., Olsson, R. T., \& Berglund, L. A. (2013). Cellulose nanofibers decorated with magnetic nanoparticles-synthesis structure and use in magnetized high toughness membranes for a prototype loudspeaker. Journal Materials Chemistry C, 1, 7963-7972.

Gea, S., Bilotti, E., Reynolds, C. T., Soykeabkeaw, N., \& Peijs, T. (2010). Bacterial cellulose-poly(vinyl alcohol) nanocomposites prepared by an in-situ process. Materials Letters, 64, 901-904.

Ganesan, L. M., Wirges, W., Mellinger, A., \& Gerhard, R. (2010). Piezo-optical and electro-optical behaviour of nematic liquid crystals dispersed in a ferroelectric copolymer matrix. Journal of Physics D: Applied Physics, 43, 015401.

Gutierrez, J., Fernandes, S. C. M., Mondragon, I., \& Tercjak, A. (2012). Conductive photoswitchable vanadium oxide nanopaper based on bacterial cellulose. ChemSusChem, 5, 2323-2327.

Gutierrez, J., Fernandes, S. M. C., Mondragon, I., \& Tercjak, A. (2013). Multifunctional hybrid nanopapers based on bacterial cellulose and sol-gel synthesized titanium/vanadium oxide nanoparticles. Cellulose, 20, 1301-1311.

Gutierrez, J., Tercjak, A., Argal, I., Retegi, A., \& Mondragon, I. (2012). Conductive properties of $\mathrm{TiO}_{2}$ /bacterial cellulose hybrid fibers. Journal of Colloid and Interface Science, 377, 88-93.

Gutierrez, J., Tercjak, A., \& Mondragon, I. (2010). Conductive behaviour of high $\mathrm{TiO}_{2}$ nanoparticles content of inorganic/organic nanostructured composites. Journal of American Chemical Society, 132, 873-878.

Hoenich, N. (2006). Cellulose for medical applications: Past, present, and future. Bioresources, 1, 270-280

Herod, T. E., \& Duran, R. S. (1998). Polymer-dispersed liquid crystal monolayers. Langmuir, 14, 6956-6968.

Heßler, N., \& Klemm, D. (2009). Alteration of bacterial nanocellulose structure by in situ modification using polyethylene glycol and carbohydrate additives. Cellulose, 16, 899-910.

Iguchi, M., Yamanaka, S., \& Budhiono, A. (2000). Bacterial cellulose-a masterpiece of nature's arts. Journal of Materials Science, 35, 261-270.

Jian, J., Zheng, Y., Song, W., Luan, J., Wen, X., Wu, Z., et al. (2014). In situ synthesis of silver-nanoparticles/bacterial cellulose composites for slow-released antimicrobial wound dressing. Carbohydrate Polymers, 102, 762-771.

Klemm, D., Schumann, D., Udhardt, U., \& Marsch, S. (2001). Bacterial synthesized cellulose-artifical blood vessels for microsurgery. Progress in Polymer Science. 26, 1561-1603.

Lee, K.-Y., Buldum, G., Mantalaris, A., \& Bismarck, A. (2014). More than meets the eye in bacterial cellulose: Biosynthesis, bioprocessing, and applications in advanced fiber composites. Macromolecular Bioscience, 14, 10-32.

Li, X., Chen, S., Hu, W., Shi, S., Shen, W., Zhang, X., et al. (2009). In situ synthesis of CdS nanoparticles on bacterial cellulose nanofibers. Carbohydrate Polymers, 76 509-512.
Lin, S. P., Calvar, I. L., Catchmark, J. M., Liu, J. R., Demirci, A., \& Cheng, K. Ch. (2013) Biosynthesis, production and applications of bacterial cellulose. Cellulose, 20, 2191-2219.

Liu, Ch., Yang, D., Wang, Y., Shi, J., \& Jiang, Z. (2012). Fabrication of antimicrobial bacterial cellulose- $\mathrm{Ag} / \mathrm{AgCl}$ nanocomposite using bacteria as versatile biofactory. Journal of Nanoparticle Research, 14, 1084-1089.

Marins, J. A., Soares, B. G., Dahmouche, K., Ribeiro, S. J. L., Barud, H., \& Bonemer, D. (2011). Structure and properties of conducting bacterial cellulose-polyaniline nanocomposites. Cellulose, 18, 1285-1294.

Olsson, R. T., Azizi Samir, M. A. S., Salazar-Alvarez, G., Belova, L., Stroem, V., Berglund, L. A., et al. (2010). Making flexible magnetic aerogels and stiff magnetic nanopaper using cellulose nanofibrils as templates. Nature Nanotechnology, 5, 584-588

Qiu, K., \& Netravali, A. N. (2014). A review of fabrication and applications of bacterial cellulose based nanocomposites. Polymer Reviews, 54, 598-626.

Quero, F., Nogi, M., Yano, H., Abdulsalami, K., Holmes, S. M., Sakakini, B. H., et al. (2010). Optimization of the mechanical performance of bacterial cellulose/poly(l-lactic) acid composites. ACS Applied Materials E Interfaces, 2, 321-330.

Pircher, N., Veigel, S., Aigner, N., Nedelec, J. M., Rosenau, T., \& Liebner, F. (2014). Reinforcement of bacterial cellulose aerogels with biocompatible polymers. Carbohydrate Polymers, 111, 505-513.

Shaha, N., Ul-Islama, M., Khattaka, W. A., \& Park, J. K. (2013). Overview of bacterial cellulose composites: A multipurpose advanced material. Carbohydrate Polymers, 98, 1585-1598.

Shi, Z., Zhang, Y., Phillips, G. O., \& Yang, G. (2014). Utilization of bacterial cellulose in food. Food Hydrocolloids, 35, 539-545.

Sumana, G., \& Raina, K. K. (2005). Electro-optic properties of aligned polysiloxane dispersed ferroelectric liquid crystal composite thin films. Current Applied Physics, 5, 277-284.

Tercjak, A., Serrano, E., \& Mondragon, I. (2006). Thermally reversible materials based on thermosetting systems modified with polymer dispersed liquid crystal for optoelectronic applications. Polymers for Advanced Technologies, 17 835-840.

Tercjak, A., García, I., \& Mondragon, I. (2008). Liquid crystal alignment in electro-responsive nanostructured thermosetting materials based on block copolymer dispersed liquid crystal. Nanotechnology, 19, 275701.

Tercjak, A., Gutierrez, J., Barud, H. S., Domeneguetti, R. R., \& Ribeiro, S. J. L. (2015). Nano- and macroscale structural and mechanical properties of in situ synthesized bacterial cellulose/PEO-b-PPO-b-PEO biocomposites. ACS Applied Materials E Interfaces, 7, 4142-4159.

Tercjak, A., Gutierrez, J., \& Mondragon, I. (2011). Conductive properties of photoluminescence Au/PS-b-PEO inorganic/organic hybrids containing nematic liquid crystals. Journal of Physical Chemistry C, 115, 1643-1648.

Tercjak, A., Gutierrez, J., Ocando, C., \& Mondragon, I. (2010). Conductive properties of switchable photoluminescence thermosetting systems based on liquid crystals. Langmuir, 26, 4296-4302.

Tercjak, A., Gutierrez, J., Ocando, C., Peponi, L., \& Mondragon, I. (2009). Thermoresponsive inorganic/organic hybrids based on conductive $\mathrm{TiO}_{2}$ nanoparticles embedded in poly(styrene-b-ethylene oxide) block copolymer dispersed liquid crystals. Acta Materialia, 57, 4624-4631.

Yano, H., Sugiyama, J., Nakagaito, A. N., Nogi, M., Matsuura, T., Hikita, M., et al (2005). Optically transparent composites reinforced with networks of bacterial nanofibers. Advanced Materials, 17, 153-155

Yu, H. Y., Qin, Z. Y., Yan, Ch. F., \& Yao, J. M. (2014). Green nanocomposites based on funcionalized cellulose nanocrystals. A study on the relationship between interfacial interaction and property enhancement. ACS Sustainable Chemistry $\mathcal{E}$ Engineering, 2, 875-896.

Zhang, Y., Nypelö, T., Salas, C., Arboleda, J., Hoeger, I. C., \& Rojas, O. J. (2013). Cellulose nanofibrils: From strong materials to bioactive surfaces. Journal of Renewable Materials, 1, 195-211.

Zhou, J., Collard, D. M., Park, J. O., \& Srinivasarao, M. (2002). Control of the anchoring behavior of polymer-dispersed liquid crystals: Effect of branching in the side chains of polyacrylates. Journal of American Chemical Society, 124 9980-9981.

Zhou, Q., Malm, E., Nilsson, H., Larsson, P. T., Iversen, T., Berglund, L. A., et al. (2009). Nanostructured biocomposites based on bacterial cellulosic nanofibers compartmentalized by a soft hydroxyethylcellulose matrix coating. Soft Matter, 5, 4124-4130

Zhu, H., Fang, Z., Preston, C., Li, Y., \& Hu, L. (2014). Transparent paper: Fabrications, properties, and device applications. Energy \& Environmental Science, 7 , 269-287. 